\title{
New constraints on the membership of the T dwarf S Ori 70 in the $\sigma$ Orionis cluster
}

\author{
M. R. Zapatero Osorio ${ }^{1}$, V. J. S. Béjar ${ }^{1}$, G. Bihain ${ }^{1,2}$, E. L. Martín ${ }^{1,3}$, R. Rebolo ${ }^{1,2}$, I. Villó-Pérez ${ }^{4}$, A. Díaz-Sánchez ${ }^{5}$, \\ A. Pérez Garrido ${ }^{5}$, J. A. Caballero ${ }^{6}$, T. Henning ${ }^{6}$, R. Mundt ${ }^{6}$, D. Barrado y Navascués ${ }^{7}$, and C. A. L. Bailer-Jones ${ }^{6}$ \\ 1 Instituto de Astrofísica de Canarias, 38200 La Laguna, Tenerife, Spain \\ e-mail: mosorio@iac.es \\ 2 Consejo Superior de Investigaciones Científicas, Spain \\ 3 University of Central Florida, Department of Physics, PO Box 162385, Orlando, FL 32816, USA \\ 4 Departamento de Electrónica, Universidad Politécnica de Cartagena, 30202 Cartagena, Spain \\ 5 Departamento de Física Aplicada, Universidad Politécnica de Cartagena, 30202 Cartagena, Spain \\ ${ }^{6}$ Max-Planck Institut für Astronomie, Königstuhl 17, 69117 Heidelberg, Germany \\ 7 LAEFF-INTA, PO 50727, 28080 Madrid, Spain
}

Received 2 September 2007 / Accepted 10 October 2007

\section{ABSTRACT}

\begin{abstract}
Aims. The nature of S Ori 70 (S Ori J053810.1-023626), a faint mid-T type object found towards the direction of the young $\sigma$ Orionis cluster, is still under debate. We intend to find out whether it is a field brown dwarf or a 3-Myr old planetary-mass member of the cluster.

Methods. We report on near-infrared $J H K_{\mathrm{s}}$ and mid-infrared [3.6] and [4.5] IRAC/Spitzer photometry recently obtained for S Ori 70. The new near-infrared images (taken $3.82 \mathrm{yr}$ after the discovery data) allowed us to derive the first proper motion measurement for this object.

Results. The colors $\left(H-K_{\mathrm{s}}\right),\left(J-K_{\mathrm{s}}\right)$ and $K_{\mathrm{s}}-[3.6]$ appear discrepant when compared to T4-T7 dwarfs in the field. This behavior could be ascribed either to a low-gravity atmosphere or to an atmosphere with a metallicity that is significantly different than solar. The small proper motion of S Ori $70\left(11.0 \pm 5.9\right.$ mas yr$\left.^{-1}\right)$ indicates that this object is farther away than expected if it were a single field $\mathrm{T}$ dwarf lying in the foreground of the $\sigma$ Orionis cluster. Our measurement is consistent with the proper motion of the cluster within $1.5 \sigma$ the astrometric uncertainty.

Conclusions. Taking into account both S Ori 70's proper motion and the new near- and mid-infrared colors, a low-gravity atmosphere remains as the most likely explanation for our observations. This supports S Ori 70's membership in $\sigma$ Orionis, with an estimated mass in the interval 2-7 $M_{\text {Jup }}$, in agreement with our previous derivation.
\end{abstract}

Key words. stars: low mass, brown dwarfs - stars: pre-main-sequence - open clusters and associations: individual: $\sigma$ Orionis

\section{Introduction}

Knowledge of the initial mass function is crucial for understanding the formation processes of stars, brown dwarfs, and freefloating planetary-mass objects. Whether and where there is a limit on the creation of objects by direct collapse and fragmentation of molecular clouds has become one of the major goals in the study of very young populations. Planetary-mass candidates with masses in the interval 3-13 Jovian masses $\left(M_{\text {Jup }}\right)$ have been found in various star-forming regions (e.g., Lucas \& Roche 2000; Zapatero Osorio et al. 2000; Chauvin et al. 2004; Lucas et al. 2005; Luhman et al. 2005; Jayawardhana \& Ivanov 2006; Allers et al. 2006; González-García et al. 2006; Caballero et al. 2007). These objects are mostly free-floating, but in a few cases they appear as wide companions to young brown dwarfs or lowmass stars.

S Ori 70 (S Ori J053810.1-023626) is the coolest freefloating, planetary-mass candidate so far reported in the literature. It was discovered by Zapatero Osorio et al. (2002a) and lies in the direction of the $\sigma$ Orionis cluster (352 pc and 1-8 Myr, with a best estimate at $3 \mathrm{Myr}$; Perryman et al. 1997; Oliveira et al. 2002; Zapatero Osorio et al. 2002b;
Sherry et al. 2004). The spectral type of S Ori 70 was determined at $\mathrm{T} 5.5 \pm 1.0$ from molecular indices measured over near-infrared $H$ - and $K$-band low-resolution spectra. Martín \& Zapatero Osorio (2003) obtained an intermediate-resolution spectrum from 1.17 to $1.37 \mu \mathrm{m}$ ( $J$-band), in which the K I doublet at $1.25 \mu \mathrm{m}$ was detected. After comparison with theoretical spectra from Allard et al. (2001), the authors inferred an effective temperature and surface gravity of $T_{\text {eff }}=1100_{-100}^{+200} \mathrm{~K}$ and $\log g=3.5 \pm 0.5 \mathrm{~cm} \mathrm{~s}^{-2}$, in agreement with the expectations for a few megayears-old T dwarf. State-of-the-art evolutionary models (Chabrier \& Baraffe 2000; Burrows et al. 1997; Baraffe et al. 1998 ) yield a mass of $3_{-1}^{+5} M_{\text {Jup }}$ if S Ori 70's very young age is finally confirmed.

Burgasser et al. (2004), in contrast, have raised doubts about the low-gravity atmosphere and true cluster membership of S Ori 70. Based on the supposed similarity of the observed spectra to field T6-T7 dwarfs, these authors argue that the S Ori object is "an old, massive field brown dwarf lying in the foreground of the $\sigma$ Orionis cluster". However, this work relies on low signal-to-noise ratio data. Better quality photometry and spectra are needed to assess the true nature of this candidate. 
Table 1. Log of near-infrared observations of S Ori 70.

\begin{tabular}{llcccc}
\hline \hline Telescope & Instrument & $\begin{array}{c}\text { Field of view } \\
\left(\operatorname{arcmin}^{2}\right)\end{array}$ & $\begin{array}{c}\text { Pixel } \\
(\operatorname{arcsec})\end{array}$ & Observing dates & $\begin{array}{c}\text { Exposure time } \\
(\mathrm{s})\end{array}$ \\
\hline $3.5 \mathrm{~m}$ CAHA & Omega-2000 & 225 & 0.45 & 2005 Oct. 22 $(\mathrm{JH}), 2005$ Oct 25 $\left(K_{\mathrm{s}}\right)$ & $3600(J), 6000(H), 7200\left(K_{\mathrm{s}}\right)$ \\
$10 \mathrm{~m}$ KeckII & NIRSPEC & 0.59 & 0.18 & 2005 Oct. 26 $\left(J K^{\prime}\right)$ & $405(J), 270\left(K^{\prime}\right)$ \\
\hline
\end{tabular}

Table 2. Near-infrared (2MASS photometric system) and IRAC/Spitzer photometry of S Ori 70.

\begin{tabular}{lccccccc}
\hline \hline Telescope & $\begin{array}{c}J \\
(\mathrm{mag})\end{array}$ & $\begin{array}{c}H \\
(\mathrm{mag})\end{array}$ & $\begin{array}{c}K_{\mathrm{s}} \\
(\mathrm{mag})\end{array}$ & $\begin{array}{c}{[3.6]} \\
(\mathrm{mag})\end{array}$ & $\begin{array}{c}{[4.5]} \\
(\mathrm{mag})\end{array}$ & $\begin{array}{c}H-K_{\mathrm{s}} \\
(\mathrm{mag})\end{array}$ & $\begin{array}{c}J-K_{\mathrm{s}} \\
(\mathrm{mag})\end{array}$ \\
\hline 3.5 m CAHA & $19.98 \pm 0.06$ & $20.07 \pm 0.07$ & $19.60 \pm 0.08$ & $\ldots$ & $\ldots$ & $+0.48 \pm 0.11$ & $+0.38 \pm 0.10$ \\
Keck II & $19.96 \pm 0.07$ & $\ldots$ & $19.58 \pm 0.07$ & $\ldots$ & $\ldots$ & $\ldots$ & $+0.38 \pm 0.10$ \\
Spitzer & $\ldots$ & $\ldots$ & $\ldots$ & $18.62 \pm 0.30$ & $17.19 \pm 0.15$ & $\ldots$ & $\ldots$ \\
\hline
\end{tabular}

Here we present astrometric measurements, IRAC/Spitzer data, and $J H K_{\mathrm{s}}$ photometry for S Ori 70 . We find that this object has unexpected colors for its spectral classification, which is measured in the range T4.5-T7 with a best estimate at T6. We ascribe this to a low-gravity atmosphere, with a different metallicity being an alternative, but less likely, explanation.

\section{Observations}

\subsection{Near-infrared photometry}

S Ori 70 was observed in $J, H$, and $K_{\mathrm{s}}$ broad-band filters with a prime focus wide-field camera Omega-2000 $(2048 \times 2048$ pixels; Bailer-Jones et al. 2000) on the $3.5 \mathrm{~m}$ telescope at the Calar Alto (CAHA) Observatory. We also imaged S Ori 70 with the NIRSPEC-3 (similar to $J$, see Fig. A.1) and $K^{\prime}$ filters and the slit-viewing camera $(256 \times 256$ pixels $)$ of the near-infrared spectrometer NIRSPEC (McLean et al. 2000) on the Keck II telescope (Hawai'i). The observing log containing instrumental information, dates of observations, and exposure times per filter is provided in Table 1 . Images ( $J$-band) of the T4.5 spectral standard dwarf 2MASS J05591914-1404488 (J0559-14 from now on) were collected with NIRSPEC immediately after S Ori 70; total exposure time was $27 \mathrm{~s}$. During both the CAHA and Keck observations, the weather was photometric and the average seeing was 0.' 8 (CAHA) and 0.'5 (Keck) in the $K$-band. Raw data were reduced in the standard fashion and included sky subtraction, flat-fielding, and combination of individual frames.

We performed point-spread-function (PSF) photometry using IRAF ${ }^{1}$. The CAHA and Keck instrumental magnitudes were transformed into observed magnitudes using between 1 and 33 2MASS sources (depending on the filter) that were present within the fields of view. The dispersion of the photometric zero point is \pm 0.05 mag in all bands. The T dwarfs exhibit complex spectral energy distributions within the near-infrared. To account for this effect and to transform the CAHA and Keck magnitudes into the 2MASS photometric system, we applied the following correction factors: $-0.04 \mathrm{mag}(J),+0.02 \mathrm{mag}(H)$, and $+0.11 \mathrm{mag}\left(K_{\mathrm{s}}\right)$ for CAHA data, $+0.02 \mathrm{mag}(J)$, and $-0.03 \mathrm{mag}$ $\left(K^{\prime}\right)$ for Keck data. These were obtained via the integration of observed near-infrared spectra of both main-sequence stars

${ }^{1}$ IRAF is distributed by National Optical Astronomy Observatories, which is operated by the Association of Universities for Research in Astronomy, Inc., under contract to the National Science Foundation, USA. of different spectral types, as well as T dwarfs (Pickles 1998; Leggett et al. 2002; Geballe et al. 2002), convolved with the Omega-2000, NIRSPEC, and 2MASS filter passbands and atmospheric transmission curves of the observatories. All filter passbands are plotted in Fig. A.1. Corrections are expected to be larger for the $J$ and $K$ filters than for the $H$-band. Nevertheless, we note that color corrections are similar or slightly smaller than the measured photometric uncertainties. CAHA and Keck magnitudes (2MASS photometric system) of S Ori 70 are reported in Table 2. As a test of consistency, we also calibrated the Keck $J$ magnitude of S Ori 70 using the observations of J0559-14 (T4.5, any color effect should be minimal), deriving $J=19.89 \pm 0.05 \mathrm{mag}$, which coincides within the observed errors with the values given in Table 2 . The excellent agreement in $J$ and $K$ between the CAHA and Keck data indicates that no strong systematic errors are affecting the measurements. Our data suggests that $\mathrm{S}$ Ori 70 does not show significant photometric variability over a few days $(\leq 0.1 \mathrm{mag})$.

\subsection{Mid-infrared photometry}

We derived mid-infrared photometry using public mosaic images available from the Spitzer Space Telescope Data Archive, taken on 2004 October 9 under the Spitzer Guaranteed Time Observation program \#37. Hernández et al. (2007) provides information on data aquisition, exposure times, and data reduction. We downloaded mosaic images in the four channels (3.6, 4.5, 5.8, and $8.0 \mu \mathrm{m}$ ) of the IRAC instrument (Fazio et al. 2004) using the Leopard software. S Ori 70 is only detected in the [3.6] and [4.5] bands with signal-to-noise ratios of 9 and 16 in the peak flux. We performed aperture photometry on various single, bright sources near S Ori 70 using daophot in IRAF, with an aperture radius of $12^{\prime \prime}$ and a background annulus extending from 12 to $24^{\prime \prime}$. For the [3.6] and [4.5] bands, we adopted daophot zero point magnitudes of 17.30 and 16.82, respectively, as described at http://ssc. spitzer. caltech. edu/archanaly/quick. phot/. We derived the PSF from the brighter sources and applied this to determine PSF photometry for S Ori 70. Results are reported in Table 2.

\subsection{Proper motion}

We determined the proper motion of S Ori 70 via the comparison of its position with respect to four common point-like sources in various near-infrared images obtained at different epochs. As 
Table 3. Proper motion of S Ori 70.

\begin{tabular}{lcc}
\hline \hline Telescope & $\begin{array}{c}\mu_{\alpha} \cos \delta \\
\left(\mathrm{mas} \mathrm{yr}^{-1}\right)\end{array}$ & $\begin{array}{c}\mu_{\delta} \\
\left(\mathrm{mas} \mathrm{yr}^{-1}\right)\end{array}$ \\
\hline 3.5 m CAHA & $+9.9 \pm 2.6$ & $+6.0 \pm 5.6$ \\
Keck II & $+9.8 \pm 4.2$ & $+3.7 \pm 4.2$ \\
Average & $+9.8 \pm 3.4$ & $+5.0 \pm 4.9$ \\
\hline
\end{tabular}

a starting point for the astrometric analysis, we used the $K_{\mathrm{s}}$ band frame shown in Fig. 1 of Zapatero Osorio et al. (2002a), which was obtained on 2001 December 29 with the Keck I telescope and has an average seeing of 0.8 and a field of view of $32^{\prime \prime} \times 32^{\prime \prime}$. The angular resolution was 0 ! $^{\prime} 15 \mathrm{pix}^{-1}$. The three recent CAHA $J H K_{\mathrm{s}}$ images and the Keck $J$ and $K^{\prime}$ frames reported here act as the second-epoch astrometric data, hence, the time interval spanned by the available observations is $3.82 \mathrm{yr}$. Our proper motion measurements are shown in Table 3 using the CAHA and Keck data separately, as well as the combined result. The associated uncertainty is derived from the dispersion of the four reference stars around null motion and the dispersion of the proper motion measurements obtained for S Ori 70 using the various images $\left(J H K_{\mathrm{S}} K^{\prime}\right)$ available per epoch.

\section{Discussion}

The low proper motion of S Ori $70\left(\mu=11.0 \pm 5.9\right.$ mas $\left.\mathrm{yr}^{-1}\right)$ makes it unlikely that it is a nearby $(\leq 30 \mathrm{pc}) \mathrm{T}$ dwarf. On the one hand, we compared our measurement with the motion of 192 Hipparcos stars (Perryman et al. 1997) within a radius of $15^{\circ}$ around $\sigma$ Orionis and at a distance between 80 and $130 \mathrm{pc}$, which is the distance interval expected for S Ori 70 if it were a field, single T6 dwarf. About $70 \%$ of the Hipparcos stars show larger motion than S Ori 70, suggesting that the S Ori object is located farther away. On the other hand, our measurement is consistent (within $1.5 \sigma$ ) with the proper motion of the 09.5V-B0.5V-type star $\sigma$ Orionis $\mathrm{AB}$, which is the most massive member of the cluster of the same name. We note, however, that the relative motion of the Orion OB association is directed away from the Sun (de Zeeuw et al. 1999). This makes it very hard to detect cluster members via proper motion analysis. On the contrary, radial velocity studies can be more discriminating (Jeffries et al. 2006), but the extreme faintness of S Ori 70 prevents any accurate radial velocity measurement with current instrumentation.

\subsection{Color-color diagrams}

Color-color diagrams are depicted in Fig. 1. To put S Ori 70 into context, we included $J H K_{\mathrm{S}}$ data of more than 100 field T-dwarfs and IRAC/Spitzer photometry of 36 field T-dwarfs compiled from the literature (Knapp et al. 2004; Tinney et al. 2005; Patten et al. 2006; Burgasser et al. 2006a; Artigau et al. 2006; Mugrauer et al. 2006; Leggett et al. 1999, 2002, 2007; Liu et al. 2007; Luhman et al. 2007; Looper et al. 2007). All near-infrared colors were conveniently transformed into the 2MASS photometric system using equations quoted in Stephens \& Leggett (2004), which are appropriate for ultracool dwarfs.

The location of S Ori 70 in Fig. 1 is challenging since this object appears as an outlier, particularly when the $K$-band magnitude is involved. Two field dwarfs lie near it in the near-infrared color-color panels: 2MASS J00501994-3322402 (Tinney et al. 2005), whose photometric errors are quite large, and 2MASS J13243559+6358284 (Looper et al. 2007). The latter object is widely discussed by Looper et al. (2007) in terms of binarity and low-gravity atmosphere. We applied the criterion defined by Covey et al. (2007, Eq. (2)) to distinguish objects with photometric properties deviating from the properties typical of field dwarfs and found that S Ori 70 lies more than $2 \sigma$ away from the near-infrared sequence defined by the field T-type brown dwarfs. In the mid-infrared wavelengths, the photometry of S Ori 70 deviates from the field at a $1-2 \sigma$ level. The Covey et al. equation takes into account the color uncertainties of S Ori 70 and the width of the field distribution.

As compared to T4-T7 field dwarfs, S Ori 70 presents redder $\left(H-K_{\mathrm{s}}\right),\left(J-K_{\mathrm{s}}\right)$, and [3.6]-[4.5] colors and a bluer $K_{\mathrm{s}}-$ [3.6] index than expected for its spectral type ( T6). However, the $(J-H)$ index is similar to that of T5-T6 field dwarfs. The $K$-band reddish nature of S Ori 70 is also apparent in its lowresolution $H K$ spectrum. Figure 4 of Burgasser et al. (2004) shows these data along with the spectrum of the field T6.5 2MASS J10475385+2124234. Both spectra were obtained with similar instrumentation and are normalized to unity at $1.57 \mu \mathrm{m}$. While the field dwarf matches the $H$-band region of S Ori 70 reasonably well, it underestimates the flux at $K$-band, supporting the redder $\left(H-K_{\mathrm{S}}\right)$ index of S Ori 70. Burgasser et al. (2004) argue that this may be indicative of a "lower surface gravity for S Ori 70 relative to 2MASS J10475385+2124234".

Multiplicity cannot explain the observed photometric properties of S Ori 70. We artificially produced near-infrared colors of $\mathrm{L}-\mathrm{T}$ and $\mathrm{T}-\mathrm{T}$ pairs using the absolute magnitudes provided by Liu et al. (2006). None of the combinations were able to reproduce our observations.

The comparison of our data to theory is shown in the right panel of Fig. 1. The models depicted are those of Tsuji et al. (2004), but in our analysis we also employed cloudless models by Marley et al. (2002) and Burrows et al. (2006), obtaining similar results. The agreement between the models and the field dwarf observations is reasonably good. The great majority of the mid- and late-T dwarfs lie within the $\log g=4.0$ and 5.5 dex tracks, as expected for "old" dwarfs in the solar neighborhood. This is also consistent with the recent results of the spectral fitting work by Burgasser et al. (2006b). These authors employed models by Burrows et al. (2006).

The near-infrared photometry of S Ori 70 and current stateof-the-art theory of ultracool dwarfs indicate that this object may have a lower-gravity atmosphere than similarly classified $\mathrm{T}$ dwarfs in the solar vicinity. Because of the different pressure and density conditions at which $\mathrm{H}_{2}, \mathrm{CH}_{4}$, and $\mathrm{CO}$ absorptions are produced, low-gravity objects tend to be brighter at $K$ and redder in all near-infrared and [3.6]-[4.5] colors than comparable high-gravity objects (see discussions and figures in Knapp et al. 2004; Patten et al. 2006; Leggett et al. 2007; Liebert \& Burgasser 2007). This is what we qualitatively observe in S Ori 70.

From the right panel of Fig. 1 and using the solar-metallicity models by Tsuji et al. (2004), we derive $\log g \sim 3.0 \mathrm{dex}$ and $T_{\text {eff }} \sim 1000-1100 \mathrm{~K}$. This is consistent with previous results from the spectral fitting analysis of low- and intermediate-resolution near-infrared spectra: $T_{\text {eff }} \sim 800_{-100}^{+200} \mathrm{~K}$, and $\log g \sim 4.0 \pm 1.0$ dex (Zapatero Osorio et al. 2002a), $T_{\text {eff }} \sim 1100_{-100}^{+200} \mathrm{~K}$, and $\log g \sim 3.5 \pm 0.5$ dex (Martín \& Zapatero Osorio 2003), respectively. These authors compared observations to theoretical data computed by Allard et al. (2001). Recently, Liu et al. (2007) have quantified the sensitivity of near-infrared spectra with $T_{\text {eff }} \sim 700-900 \mathrm{~K}$ to changes in metallicity and surface gravity using a different grid of synthetic spectra by 

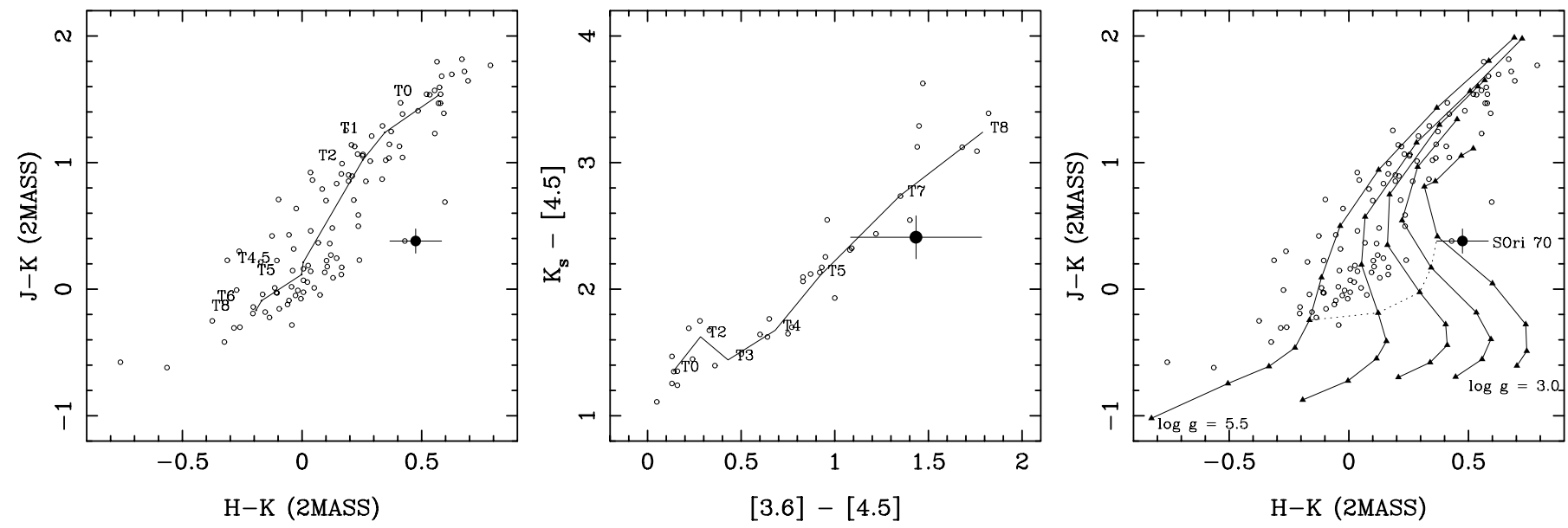

Fig. 1. Color-color diagrams of S Ori 70 (solid circle) and field T-dwarfs (open circles). For clarity, only the error bars of S Ori 70 are plotted. In the left and middle panels, the sequence defined by the field brown dwarfs is displayed with a solid line. Spectral types are indicated. The right panel shows the solar-metallicity models by Tsuji et al. (2004) superimposed onto the data. Gravities of $\log g=3.0,3.5,4.0,4.5$, and $5.5 \mathrm{~cm} \mathrm{~s}^{-2}$ are depicted with solid lines. The triangles along each model curve are in $T_{\text {eff }}$ invervals of $100 \mathrm{~K}$, ranging from 700 (bottom) to $1500 \mathrm{~K}$ (log $g=3.0$ ), $1400 \mathrm{~K}(\log g=3.5), 1600 \mathrm{~K}(\log g=4.0$ and 4.5$)$, and $1700 \mathrm{~K}(\log g=5.5)$. The $\log g=5.0$ track is not plotted for clarity. The dotted line connects the $T_{\text {eff }}=1100 \mathrm{~K}$ points in each model.

Burrows et al. (2006). S Ori 70 is brighter at $K$ than is at $J$ or $H$ by a factor of $\sim 1.4$. Table 5 and Fig. 4 of Liu et al. (2007) suggest that $\log g$ is thus lower than the field by about $1.0 \mathrm{dex}$, in agreement with previous determinations. We caution that current state-of-the-art synthetic spectra do not provide detailed fits to the observed data (e.g., Burrows et al. 2006). Therefore, any quantitative result derived from the direct comparison of observations to models awaits further confirmation. In contrast, for a given temperature qualitative predictions on the atmospheric relative behavior as a function of gravity and metal content can be more reliable.

Metallicity might also be an issue. The $\sigma$ Orionis cluster has solar abundance $([\mathrm{Fe} / \mathrm{H}]=0.0 \pm 0.1 \mathrm{dex}$; Caballero 2006); we do not expect any metallicity effect when comparing cluster members to the field. From theoretical considerations, the effects of increasing abundance and decreasing gravity on the near-infrared spectra of cool T dwarfs are similar. From Fig. 20 of Burrows et al. (2006), which shows $(J-K)$ against $T_{\text {eff for }}$ various gravities and metal abundances, we infer a metallicity of $[\mathrm{Fe} / \mathrm{H}] \sim+0.5$ dex for S Ori 70 if its reddish effect was all due to metallicity. A similarly rich metal content is obtained from Liu et al. (2007). Nevertheless, the super-solar metallicity explanation, although possible, seems unlikely. On the one hand, the metallicity distribution of $\mathrm{F}, \mathrm{G}$, and $\mathrm{K}$ dwarf stars in the solar neighborhood peaks at around $[\mathrm{Fe} / \mathrm{H}]=0.0$ dex and extends roughly up to $+0.5 \mathrm{dex}$; less than $\sim 10 \%$ of the stars are more metal-rich than +0.3 dex (Valenti et al. 2005; Santos et al. 2005; Boone et al. 2006). On the other hand, the IRAC/Spitzer data of the S Ori object do not support the high metallicity case. Burgasser et al. (2006b) and Liebert \& Burgasser (2007) have demonstrated 2MASS J12373919+6526148 (T6.5) and 2MASS J09373487+2931409 (T6p) to be old, high surface gravity brown dwarfs with sub-solar abundance. These field dwarfs display [3.6] - [4.5] colors that are slightly redder (by $0.15 \mathrm{mag}$ ) than expected for their assigned spectral types. In contrast to what could be inferred from the near-infrared colors, this would indicate that S Ori 70 is a low-metallicity T dwarf. Thus, a low-gravity atmosphere remains as the most likely explanation for the observed photometry of S Ori 70.
No obvious infrared flux excesses are detectable in the IRAC/Spitzer [3.6] and [4.5] bands, suggesting that there is no envelope or disk around S Ori 70 emitting intensively at these wavelengths. A positive detection would have provided strong evidence for its youth. However, we note that disks around young, low-mass brown dwarfs (close to the deuteriumburning mass limit) are seen at wavelengths longer than $5 \mu \mathrm{m}$ (Luhman et al. 2005; Caballero et al. 2007; Zapatero Osorio et al. 2007), while the observed fluxes in the near-infrared up to $5 \mu \mathrm{m}$ are photospheric in origin. The public [5.8]- and [8.0]band IRAC/Spitzer images are not conclusive for S Ori 70.

\subsection{Color-magnitude diagram}

The photometric sequence of $\sigma$ Orionis substellar members, including S Ori 70, is shown in the $J$ vs. $J-[3.6]$ color-magnitude diagram of Fig. 2 (Caballero et al. 2007; Zapatero Osorio et al. 2007). This sequence follows a relatively smooth progression with increasing color down to $J \sim 20$ and $J-[3.6]=2.8 \mathrm{mag}$. The location of S Ori 70 suggests that the $J-[3.6]$ index suddenly turns toward bluer values at a nearly unchanged $J$ magnitude. A similar turnover (occurring at spectral types L7-L8, $1400-1300 \mathrm{~K}$ ) is also observed in field ultracool dwarfs. The field sequence of objects with spectral types M5.5-T8 moved to the distance of the $\sigma$ Orionis cluster is displayed in the right panel of Fig. 2 (absolute magnitudes and colors are adopted from Patten et al. 2006, and references therein). Because of their very young age, $\sigma$ Orionis low-mass stars and substellar objects are in the phase of gravitational contraction (e.g., Chabrier \& Baraffe 2000). Cluster members thus show larger size and higher luminosity than their older counterparts of related colors in the field. As seen in Fig. 2, the average cluster photometric sequence appears brighter than the field by about $1.8 \mathrm{mag}$ in the $J$-band. The dotted line in Fig. 2 (right panel) represents the field sequence normalized to the $\sigma$ Orionis locus of late-M and early-L cluster members. S Ori 70 nicely sits on the location expected for $\sigma$ Orionis T-type members.

We also compared our data to the COND and DUSTY solar metallicity evolutionary models by Chabrier \& Baraffe (2000). 

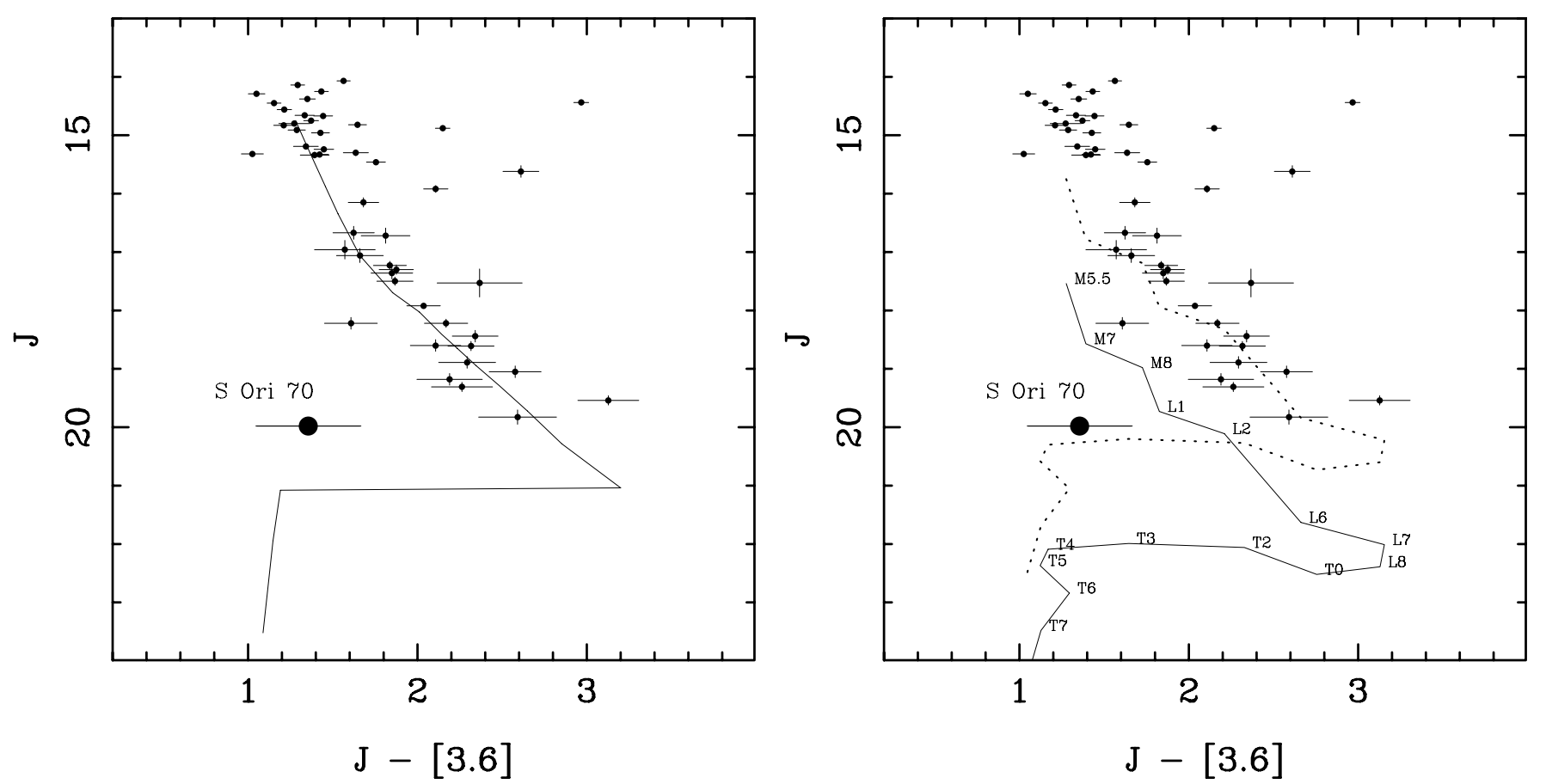

Fig. 2. Color-magnitude diagram of $\sigma$ Orionis low mass members. S Ori 70 is labeled. The 3-Myr isochrone by Chabrier \& Baraffe (2000) is overplotted onto the data as a solid line in the left panel (see text for $T_{\text {eff }}, \log L / L_{\odot}$ conversion into observables). The right panel shows the sequence of M5.5-T8 field dwarfs at the distance of the cluster (solid line, spectral types are indicated). The dotted line stands for the field sequence shifted by $-1.8 \mathrm{mag}$ in the $J$-band to match the photometric trend delineated by $\sigma$ Orionis members. Objects with $J-[3.6$ ] colors significantly redder than the cluster sequence show infrared flux excesses likely due to circum(sub)stellar disks (Caballero et al. 2007).

For the age range $1-8 \mathrm{Myr}$, substellar objects with $T_{\text {eff }}$ between 700 and $1300 \mathrm{~K}$, corresponding to the mass interval $\sim 1-6 M_{\text {Jup }}$, show $\log g=3.0-4.0$ dex, which coincides within the large error bar with the surface gravity estimation for S Ori 70.

The 3-Myr isochrone (Chabrier \& Baraffe 2000) is displayed along the photometric sequence of $\sigma$ Orionis in the left panel of Fig. 2. Theoretical surface temperatures and luminosities were converted into observed magnitudes and colors using the color-temperature-spectral type and spectral type-bolometric correction relationships given in the literature (Dahn et al. 2002; Vrba et al. 2004; Knapp et al. 2004; Patten et al. 2006). The model convincingly reproduces the cluster low-mass sequence except for the fact that S Ori 70 appears overluminous by about $1 \mathrm{mag}$, which might suggest binarity. This was also discussed in Zapatero Osorio et al. (2002a). However, there are issues that prevent us from concluding whether this object is double or whether models make wrong predictions for the lowest masses and young ages: (i) as seen from the field sequence (Fig. 2, right panel), there is a $J$-band brightening across the color turnover that theory fails to reproduce (Vrba et al. 2004; Knapp et al. 2004). (ii) The blue color turnover takes place at a roughly constant temperature in the field $(\sim 1300-1400 \mathrm{~K})$, and all colortemperature-bolometric correction transformations show a sharp change at this point; on the contrary, the evolutionary models available to us do not have a complete temperature sampling (this may explain the abrupt color reversal at the bottom of the isochrone in Fig. 2). (iii) The relations used to transform theoretical predictions into observables are obtained for high-gravity field objects. It is now known that gravity significantly impacts the near- and mid-infrared colors of $\mathrm{T}$ dwarfs (Leggett et al. 2007; Burrows et al. 2006), whereas the colors of the warmer $\mathrm{M}$ and $\mathrm{L}$ types are not as sensitive to the gravity parameter.
Transformations are thus expected to be gravity-dependent for the coolest temperatures. It becomes necessary to find a physical explanation for the $J$ brightening feature and to discover more $\sigma$ Orionis T-type, planetary-mass members for a proper comparison with evolutionary tracks.

\section{Final remarks}

New near-infrared and Spitzer photometric data of S Ori $70(\mathrm{~T} 6 \pm 1.0)$ provide evidence that this object is young. Its colors qualitatively follow the trend predicted for low-gravity atmospheres by state-of-the-art models. Its very low proper motion is consistent with membership in the $\sigma$ Orionis cluster. The mass of S Ori 70 is then estimated from substellar evolutionary models to be within the planetary regime in the range 2-7 $M_{\text {Jup }}$ (Zapatero Osorio et al. 2002a; Martín \& Zapatero Osorio 2003). This mass interval takes into account both the object's luminosity uncertainty and the most recent cluster-age determination (1-7 Myr; Sherry et al. 2004; Jeffries et al. 2006). The existence of such low-mass objects in isolation at ages below $10 \mathrm{Myr}$ is a challenging and interesting issue for the theory of formation of stars, brown dwarfs, and planets.

Acknowledgements. We are indebted to T. Tsuji and M. Marley for providing computer-ready files of their models. We also thank Sandy Leggett (referee) for useful comments that helped us improve this work. Based on observations collected at the Centro Astronómico Hispano Alemán (CAHA) at Calar Alto, operated jointly by the Max-Planck Institut für Astronomie and the Instituto de Astrofísica de Andalucía (CSIC). Some data were also obtained at the W. M. Keck Observatory, which is operated as a scientific partnership between the California Institute of Technology, the University of California, and NASA. The Observatory was made possible by the generous financial support of the W. M. Keck Foundation. We thank the Keck observing assistants and the staff in Waimea for their kind support. The authors extend special thanks to those of Hawaiian ancestry on whose sacred mountain we are privileged to be guests. This 

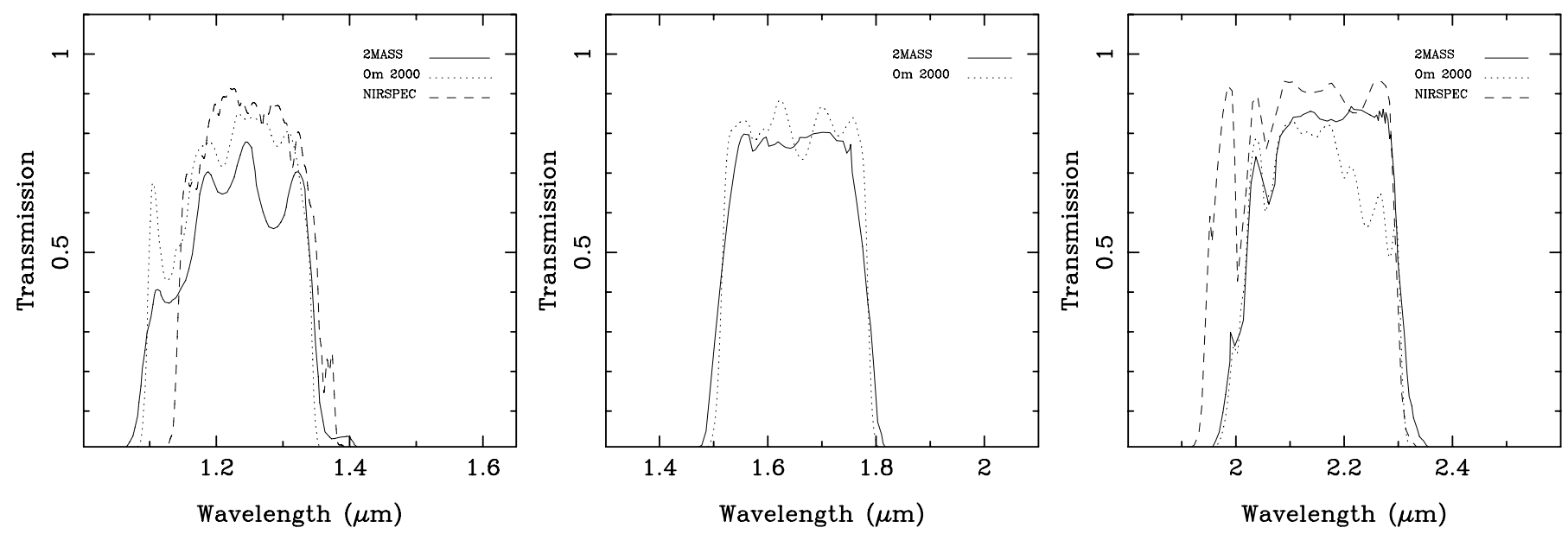

Fig. A.1. Passbands of the $J$ (left), $H$ (middle), and $K$ (right) filters used in this work convolved with the observatories atmospheric transmission curves. Labels correspond to the NIRSPEC (Keck) and Omega-2000 (CAHA 3.5 m) instruments, and 2MASS filters. Data have been taken from the observatories' web pages. The Omega-2000 filter passbands were convolved with the 2MASS atmospheric transmission curve because this information is not available on the CAHA web site.

research has made use of data products from the Two Micron All Sky Survey, which is a joint project of the University of Massachusetts and the Infrared Processing and Analysis Center/California Institute of Technology, funded by the National Aeronautics and Space Administration and the National Science Foundation. This work is based in part on archival data obtained with the Spitzer Space Telescope, which is operated by the Jet Propulsion Laboratory, California Institute of Technology under a contract with NASA. Support for this work was provided by an award issued by JPL/Caltech. Partial financial support was provided by the Spanish projects AYA2003-05355 and AYA2006-12612.

\section{Appendix A: Filter passbands}

See Fig. A.1.

\section{References}

Allard, F., Hauschildt, P. H., Alexander, D. R., Tamanai, A., \& Schweitzer, A. 2001, ApJ, 556, 357

Allers, K. N., Kessler-Silacci, J. E., Cieza, L. A., \& Jaffe, D. T. 2006, ApJ, 644, 364

Artigau, E., Doyon, R., Lafrenière, D., et al. 2006, ApJ, 651, L57

Bailer-Jones, C. A. L., Bizenberger, P., \& Storz, C. 2000, Proc. SPIE, 4008, 1305 Baraffe, I., Chabrier, G., Allard, F., \& Hauschildt, P. H. 1998, A\&A, 337, 403

Boone, R. H., King, J. R., \& Soderblom, D. R. 2006, New A. Rev., 50, 526

Burgasser, A. J., Kirkpatrick, J. A., McGovern, M. R., et al. 2004, ApJ, 604, 827 Burgasser, A. J., Geballe, T. R., Leggett, S. K., Kirkpatrick, J. D., \& Golimowski, D. A. 2006a, ApJ, 637, 1067

Burgasser, A. J., Burrows, A., \& Kirkpatrick, J. D. 2006b, ApJ, 639, 1095

Burrows, A., Marley, M., Hubbard, W. B., et al. 1997, ApJ, 491, 856

Burrows, A., Sudarsky, D., \& Hubeny, I. 2006, ApJ, 640, 1063

Caballero, J. A. 2006, Ph. D. Thesis, Univ. La Laguna (Tenerife, Spain)

Caballero, J. A., Béjar, V. J. S., Rebolo, R., et al. 2007, A\&A, 470, 903

Chabrier, G., \& Baraffe, I. 2000, ARA\&A, 38, 337

Chauvin, G., Lagrange, A.-M., Dumas, C., et al. 2004, A\&A, 425, L29

Chauvin, G., Lagrange, A.-M., Dumas, C., et al. 2005, A\&A, 438, L25

Covey, K. R., Ivezić, Z., Schlegel, D., et al. 2007, ApJ, in press

Dahn, C. C., Harris, H. C., Vrba, F. J., et al. 2002, AJ, 124, 1170

de Zeeuw, P. T., Hoogerwerf, R., de Bruijne, J. H. J., Brown, A. G. A., \& Blaauw, A. 1999, AJ, 117, 354

Fazio G. G., Hora, J. L., Allen, L. E., et al. 2004, ApJ Suppl., 154, 10

Geballe, T. R., Knapp, G. R., Leggett, S. K., et al. 2002, ApJ, 564, 466

González-García, B. M., Zapatero Osorio, M. R., Béjar, V. J. S., et al. 2006, A\&A, 460, 799
Hernández, J., Hartmann, L., Megeath, T., et al. 2007, ApJ, 662, 1067

Jayawardhana, R., \& Ivanov, V. D. 2006, Science, 313, 1279

Jeffries, R. D., Maxted, P. F. L., Oliveira, J. M., \& Naylor, T. 2006, MNRAS, 371, L6

Knapp, G., Leggett, S. K., Fan, X., et al. 2004, AJ, 127, 3553

Leggett, S. K., Toomey, D. W., Geballe, T. R., \& Brown, R. H. 1999, ApJ, 517, L139

Leggett, S. K., Golimowski, D. A., Fan, X., et al. 2002, ApJ, 564, 452

Leggett, S. K., Saumon, D., Marley, M. S., Geballe, T. R., \& Fan, X. 2007, ApJ, 655,1079

Liebert, J., \& Burgasser, A. J. 2007, Apj, 655, 522

Liu, M. C., Leggett, S. K., Golimowski, D. A., et al. 2006, ApJ, 647, 1393

Liu, M. C., Leggett, S. K., \& Chiu, K. 2007, ApJ, 1507

Looper, D. L., Kirkpatrick, J. D., \& Burgasser, A. J. 2007, AJ, 134, 1162

Lucas, P. W., \& Roche, P. F. 2000, MNRAS, 314, 858

Lucas, P. W., Roche, P. F., \& Tamura, M. 2005, MNRAS, 361, 211

Luhman, K. L., Adame, L., D’Alessio, P., et al. 2005, ApJ, 635, L93

Luhman, K. L., Patten, B. M., Marengo, M., et al. 2007, ApJ, 654, 570

Marley, M. S., Seager, S., Saumon, D., et al. 2002, ApJ, 568, 335

McLean, I. S., Wilcox, M. K., Becklin, E. E., et al. 2000, ApJ, 533, L45

Martín, E. L., \& Zapatero Osorio, M. R. 2003, ApJ, 593, L113

Mugrauer, M., Seifahrt, A., Neuhäuser, R., \& Mazeh, T. 2006, MNRAS, 373, L31

Oliveira, J. M., Jeffries, R. D., Kenyon, M. J., Thompson, S. A., \& Naylor, T. 2002, A\&A, 382, L22

Patten, B. M., Stauffer, J. R., Burrows, A., et al. 2006, ApJ, 651, 502

Perryman, M. A. C., Lindegren, L., Kovalevsky, J., et al. 1997, A\&A, 323, L49

Pickles, A. J. 1998, PASP, 110, 863

Santos, N. C., Israelian, G., Mayor, M., et al. 2005, A\&A, 437, 1127

Sherry, W. H., Walter, F. M., \& Wolk, S. J. 2004, AJ, 128, 2316

Stephens, D. C., \& Leggett, S. K. 2004, PASP, 116, 9

Tinney, C. G., Burgasser, A. J., Kirkpatrick, J. D., \& McElwain, M. W. 2005, AJ, 130,2326

Tsuji, T., Nakajima, T., \& Yanagisawa, K. 2004, ApJ, 607, 511

Valenti, J. A., \& Fischer, D. A. 2005, ApJ Suppl., 159, 141

Vrba, F. J., Henden, A. A., Luginbuhl, C. B., et al. 2004, AJ, 127, 2948

Zapatero Osorio, M. R., Béjar, V. J. S., Martín, E. L., et al. 2000, Science, 290, 103

Zapatero Osorio, M. R., Béjar, V. J. S., Martín, E. L., et al. 2002a, ApJ, 578, 536 (Paper I)

Zapatero Osorio, M. R., Béjar, V. J. S., Pavlenko, Ya., et al. 2002b, A\&A, 384, 937

Zapatero Osorio, M. R., Caballero, J. A., Béjar, V. J. S., et al. 2007, A\&A, 472, L9 